\title{
Skin Pigment Recognition using Projective Hemoglobin- Melanin Coordinate Measurements
}

\author{
Liu Yang*, Suk-Hwan Lee**, Seong-Geun Kwon***, Ha-Joo Song ${ }^{\S}$ and Ki-Ryong Kwon ${ }^{\dagger}$
}

\begin{abstract}
The detection of skin pigment is crucial in the diagnosis of skin diseases and in the evaluation of medical cosmetics and hairdressing. Accuracy in the detection is a basis for the prompt cure of skin diseases. This study presents a method to recognize and measure human skin pigment using Hemoglobin-Melanin (HM) coordinate. The proposed method extracts the skin area through a Gaussian skin-color model estimated from statistical analysis and decomposes the skin area into two pigments of hemoglobin and melanin using an Independent Component Analysis (ICA) algorithm. Then, we divide the two-dimensional (2D) HM coordinate into rectangular bins and compute the location histograms of hemoglobin and melanin for all the bins. We label the skin pigment of hemoglobin, melanin, and normal skin on all bins according to the Bayesian classifier. These bin-based HM projective histograms can quantify the skin pigment and compute the standard deviation on the total quantification of skin pigments surrounding normal skin. We tested our scheme using images taken under different illumination conditions. Several cosmetic coverings were used to test the performance of the proposed method. The experimental results show that the proposed method can detect skin pigments with more accuracy and evaluate cosmetic covering effects more effectively than conventional methods.
\end{abstract}

Keywords: Skin pigment recognition, Projective HM Coordinate, 2D location histogram

\section{Introduction}

Skin pigmentation refers to skin areas that differ in color from the regular skin. As the largest organ of the body, the skin is always influenced by some internal or external factors. The skin often reacts by modifying its constitutive pigment pattern, which allows the skin to appear different in color. When the body produces too much or little melanin, skin pigmentation disorders occur, such as blotches, uneven areas, brown patches, or spots. Additionally, this uneven pigmentation affects many people, regardless of ethnic background or skin color. Melanin is the main determinant of skin color and can also be found in the hair, the irises of the eyes, and the brain. In most cases, the human skin possesses a fairly uniform concentration of melanin. However, the concentration of melanin in each individual varies between ethnicities; this variation is expressed in the melanin-producing genes. When no melanin is found in a particular human body, this suggests a condition called albinism.

$\dagger$ Corresponding Author: Dept. of IT Convergence and Application Eng., Pukyong National Univ., Korea. (krkwon@pknu.ac.kr)

* Dept. of IT Convergence and Application Engineering, Pukyong National Univ., Korea. (yangliu.0816@hotmail.com)

** Dept. of Information Security, Tongmyong Univ., Korea. (skylee@tu.ac.kr)

*** Dept. of Electronics Engineering, KyungIl Univ., Korea. (sgkwon@kiu.ac.kr)

$\S$ Dept. of IT Convergence and Application Engineering, Pukyong National Univ., Korea. (hajoosong@pknu.ac.kr)

Received: July 24, 2015; Accepted: May 9, 2016
Hemoglobin is another factor that determines skin color, which can be made less visible by finger pressure, while bruising or bleeding under the skin increases its visibility. Hemoglobin pigment can be produced by infection, massage, electrical treatment, acne medication, allergies, exercise, or sunburn and many others, which can all cause the capillaries to dilate or break, resulting in redness. Thus, hemoglobin pigmentation is either a symptom or a disease itself, which affects the appearance and health of people.

Most unusual skin pigments are diagnosed after detection by a clinician or beautician, which is subjective and qualitative. In recent years, color measurement instruments have been developed to detect and measure skin pigment. A basic method for solving the difficult problem of detection is a visual examination. The visionbased detection of skin lesions in dermoscopic images in diagnosing ailments, such as melanoma and other pigmented lesions, are presented in various studies $[1,2]$. These algorithms focus on diagnosis and treatment of skin lesions from a medical standpoint. However, the optical instruments are expensive and the probing of color measurement instruments have greatly influenced the results.

Since 1987, investigators have started to use digital image analysis systems to detect skin pigment. Image analysis involves no direct contact with the skin and is considered safe. Image processing technology can differentiate skin pigment segments from the background and can calculate some characteristic information on the 


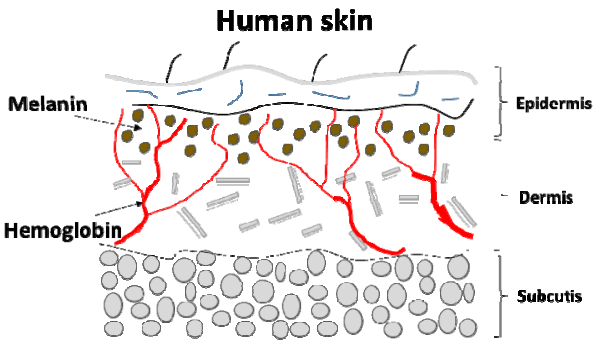

(a)

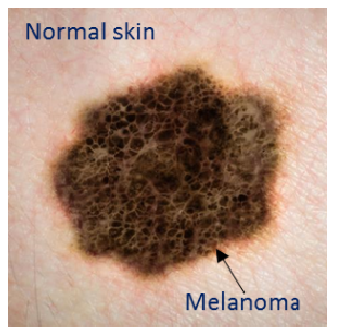

(b)

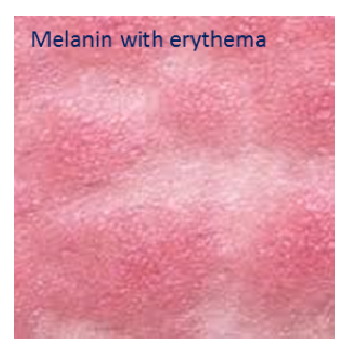

(c)

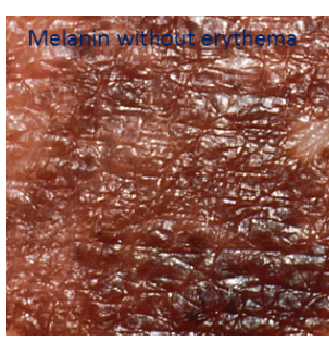

(d)

Fig. 1. (a) Schematic cross-section of the human skin tissue and examples of pigmented skin lesions; (b) melanoma; (c) melanin with erythema, and (d) melanin without erythema

skin pigment. However, an image analysis system requires high-resolution images.

Unlike skin lesions, skin pigment disorders affect the outward appearance without indication of potential health risks in most cases. Many people use cosmetics to cover these disorders for the sake of vanity. The detection and analysis of skin pigmentation are necessary to evaluate objectively the efficacy of cosmetics or whether the skin requires medical treatment. Similar to skin lesion detection, the vision-based detection approach can precisely segment a pigmented area from a normal skin area and compute the quantity of pigment measurement $[3,4]$.

In this study, we propose a scheme to detect and measure the hemoglobin and melanin levels of skin pigmentation from regular digital images. Our scheme is divided into three steps: 1) skin detection based on a Gaussian skincolor model, 2) pigment decomposition using an independent component analysis (ICA) algorithm, and 3) detection and analysis of hemoglobin and melanin by two-dimensional (2D) location histogram and Bayesian classifier. Our experiment consisted of two tests to evaluate the performance of our scheme. The first experiment tests the adaptability of our scheme under a variety of illumination conditions. The second experiment tests the measurement of cosmetic efficacy in covering pigment disorders. The experimental results show that our scheme performed satisfactorily in both tests. The recognition rate was 93\% under most conditions while achieving an acceptable processing time.

The rest of this paper is organized as follows: In Section II, skin pigmentation is defined and related studies on general skin detection and skin pigment detection are presented. In Section III, we introduce our scheme for detecting and measuring the hemoglobin and melanin of skin pigment in detail. In Section IV, the results of our two experimental tests are discussed and compared with those of previous studies. Finally, we conclude this study and provide direction for future work in Section V.

\section{Related Works}

The pigment detection should use the specific components of the color coordinate. To improve the understanding of the color coordinate of pigment, we need to evaluate the physiological structure and optical properties of the skin, which are presented in this section using the findings of Krishnawwamy et al. [5] and related works for pigment detection.

\subsection{Skin pigmentation}

Similar to the general color measurement methods, we use one component of color space to measure skin pigment. To understand the significance and measurement of color components, we need to explain the physiological structure and optical properties of the skin [5]. The color of the human skin is basically determined by two constituents, namely, melanin and hemoglobin. These constituents exist in the epidermis and dermis of the human skin as shown in Fig. 1(a). Melanin is produced by melanocytes, which partly absorb visible light and darken our skin color. Light absorption levels depend on the unit-volume of melanin, which is manifested in the skin color differences between Caucasians, Asians, and people of African descent. In blood cells, we find another natural chromophore called hemoglobin. This chromophore provides the reddish color of the skin. Aside from melanin and hemoglobin, the abnormal local composition of our skin also dictates skin color. When the level of melanin is not normal, the person may have albinism or melanoma. When the level of hemoglobin is not normal, the skin may suffer injury or become erythematic. In this study, we attempt to deconstruct skin color based on its melanin and hemoglobin components. By comparison with normal skin-color components, we can detect and quantify the melanin and hemoglobin pigments, respectively.

Figs. 1(b), 1(c), and 1(d) show examples of melanoma and melanin, pigmented skin lesions, with or without erythema, respectively. The image processing for melanin and hemoglobin components will consider two factors. The first one is the common three-dimensional (3D) color space, such as RGB and HSV, which can be reduced to a lower dimension 2D color space that makes full use of the color information and reduces calculated amount at the same time. The second factor is the physical meaning 
of the color components. Since chromophore density is quantized by color intensity, the detection of skin pigment disorders becomes no longer about yes or no question. Our proposed method can support to analyze the causes of skin pigment disorders by computer analysis using methods for medical diagnosis.

\subsection{Skin pigment detection}

Many studies presented the detection algorithms of pigmented skin lesion for medical diagnosis. Liu et al. [4] presented the skin-pigmentation-disorder detection method to assist in the diagnosis of skin cancer of melanoma or self-exam. Nugroho et al. [6] developed an image analysis for skin pigmentation to classify and quantify the eumelanin and pheomelanin types of melanin in the skin. The proposed model is based on a Monte Carlo simulation of light and skin. This model was developed by using data collected from a clinical study that involved hundreds of participants with three different skin images each. In this study, an inverse type of the model is applied to extract the information on melanin and report its concentration. The proposed approach provides an effective characterization of skin layers in determining melanin types. Madasu et al. [7] detected blotches in skin lesions by extending the fuzzy co-clustering algorithm for images (FCCI). This method further improvises by adding the texture features as a multidimensional clustering parameter, which is computed from the normalized entropy function. Clawson et al. [8] proposed an algorithm for the visual display and quantification of color asymmetry. Automatic induction and neural networks were introduced to evaluate the diagnostic capability of the features and identify the maximum correlation values. The results show that the features quantifying possible regression regions are the most indicative of color asymmetry.

In the majority of papers on pigment detection, the main application is for medical diagnosis. To obtain accurate and comparable results [9-11], most schemes need a stable illumination environment and professional dermoscopy equipment to acquire skin images. The disadvantages include limited applications and expensive instruments. As an improvement, we propose an approach that has a robust quality for illumination but does not rely on expensive professional equipment. We hope that our scheme for detection of skin pigment will have a broader range of uses.

Melanin and hemoglobin can be separated and quantized by image analysis methods. Lu et al. [12] presented a three-layer segmentation algorithm for automatic erythema detection. They detected skin regions by a histogrambased Bayesian classifier and represented skin regions in terms of melanin and hemoglobin components by ICA. Then, they identified erythema areas using feature attributes from hemoglobin and melanin components by a trained Support Vector Machine (SVM). This method can detect the pigment in low-contrast images. However, it requires more time to detect pigments and is not robust to illumination changes. Vision-based methods can recognize the disorders of pigmented skin more effectively than optical measurements [13-15]. Improved software has been developed to offset the insufficient hardware. However, the dependence on stable illumination and specialized hardware has not been sufficiently reduced [16]. We address a new method for pigment detection and quantification with robustness to illumination changes and low processing time in this study.

\subsection{Human skin detection}

With regard to the skin detection algorithm, the simplest and most obvious characteristic of skin is either its color or its texture. Fotouhi et al. [16] demonstrated a method using contourlet-based texture analysis for skin area detection. A boosted pixel-based skin detection method is used to recognize skin pixels. Skin texture features are used with contourlet texture coefficients to improve the detection performance. Candidate skin pixels are selected in all subimages and the feature vector of each patch is extracted. Multilayer perception is utilized to identify the features and classify the input images.

On the other hand, Zhengming et al. [17] proposed a scheme that uses a pixel-skipping image process instead of testing each pixel to label it as either skin or non-skin. Motivated by the efficient approach of Hsu et al. [18], a skin detection algorithm based on the RG color space has been developed. Kherchaoui and Houacine [19] combined both a statistical model of skin color and geometrical characteristics for face detection. The system presented consists of two parts: the first part detects skin color by using a statistical method and is based on a Gaussian mixture model in the $\mathrm{CbCr}$ color space; the second part concerns the detected candidate skin regions corresponding to faces.

Most skin detection methods use a relatively mature algorithm that combines color clustering with a Gaussian mixture model. The differences among these methods involve the color space and the additional algorithms for improving detection performance. These methods are shown to be effective for skin detection under limiting conditions, such as stable illumination and simple backgrounds. Without these limitations, the probability of false detections would be high. Therefore, we cluster skin pixels based on a $\mathrm{YCbCr}$ color space and minimize the influence of illumination. Moreover, different skin colors are classified according to human intuition.

\section{Proposed Skin Pigmentation Detection}

\subsection{Overview}

Our proposed method for detection of skin pigment aims 


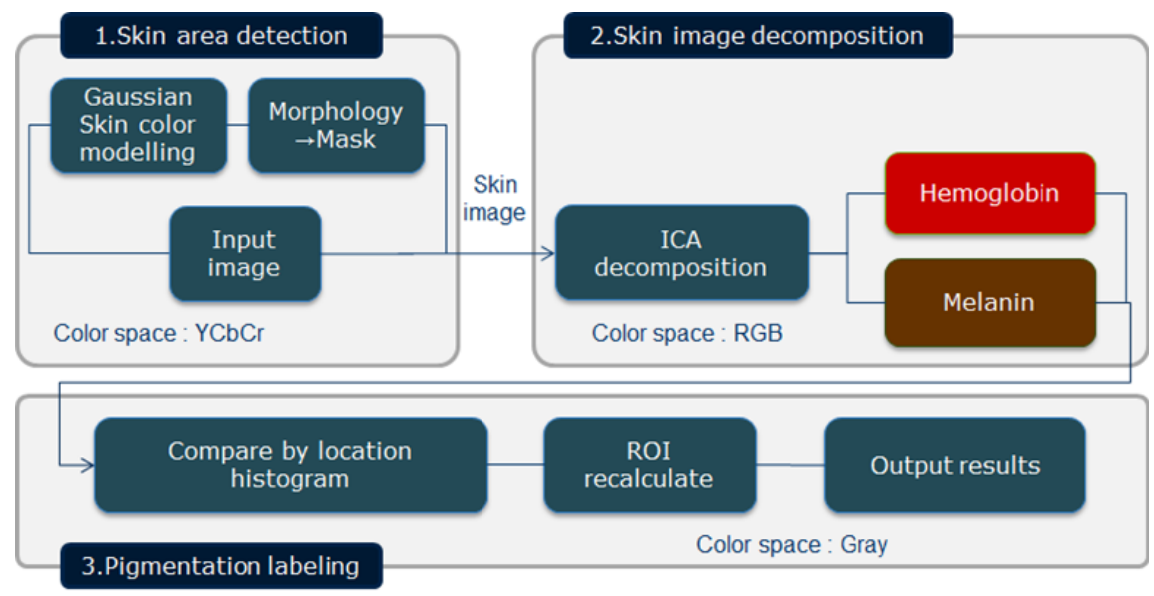

Fig. 2. The proposed skin pigment detection process

to be:

1) Resilient to illumination conditions, with a low processing time, and a high recognition rate;

2) Applicable to the medical skin diagnosis of melanoma, erythema, and other skin lesions of abnormal skin; and

3) Applicable to the cosmetic effect of skin-disorder areas in normal skin and to evaluate the cosmetic covering effect.

To consider these three aims, our proposed method consists of three steps as shown in Fig. 2. In the first step, through good clustering performance for skin color in the $\mathrm{CbCr}$ color space, we segment the skin region by using a Gaussian skin-color model and morphology processing. In the second step, we decompose the skin color into its hemoglobin and melanin components by using an ICA algorithm. Then, we can compare the respective intensity values with those of the normal skin to detect pigments. In the last step, we quantify the skin pigment intensity by using a $2 \mathrm{D}$ location histogram and we detect the pigments using a ratio of hemoglobin to melanin.

\subsection{Color space of skin pigmentation}

For skin image, several color spaces have been widely used, such as HSV, YCbCr, and normalized RGB. Their stability in different illumination environment is compared to determine which color space works better with different light intensities. As shown in Fig. 3, we make a skin sample with shadow and pigmentation and draw a green line on the sample. We can find that half part of the green line contains shadow while the other part does not, with either of them crossing a pigment spot. Then, we can make each channel density distribution of $\mathrm{YCbCr}$ color space along this yellow line as shown in Fig. 3(a). From the Y value, the pigment spot can be located; on the image, the pigment lesion value is similar to the shadow part. The shadow and pigment lesion in $\mathrm{YCbCr}$ color space are

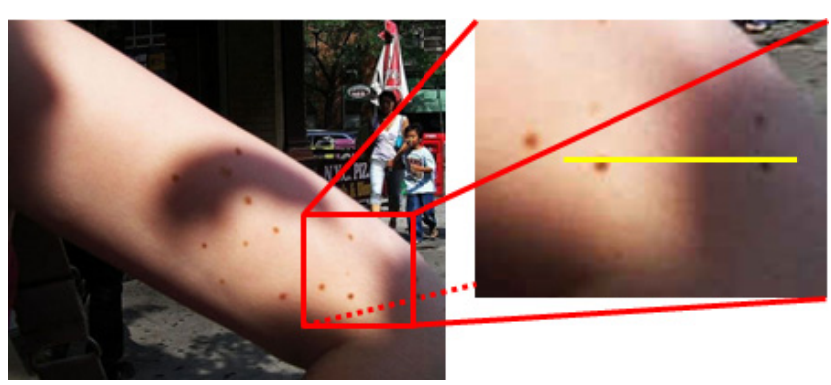

(a)

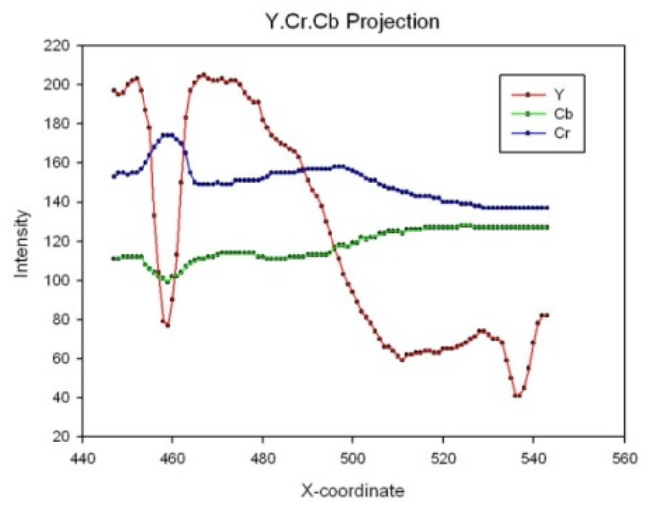

(b)

Fig. 3. (a) A sample of pigment lesion and (b) pixel density in each channel of $\mathrm{YCbCr}$

difficult to distinguish as shown in Fig. 3(b).

To extract the normal skin part, the input images are transformed into gray image, and the OTSU thresholding algorithm is used to extract part of the images. When we compare the intensity among the normal skin, the pigment lesion area, the shadow part, and hair, we found that the normal skin has the highest intensity. With its significance to the OTSU, part of the normal skin will be segmented as foreground. The OTSU will segment the image into foreground and background based on the image intensity using the following expressions. 


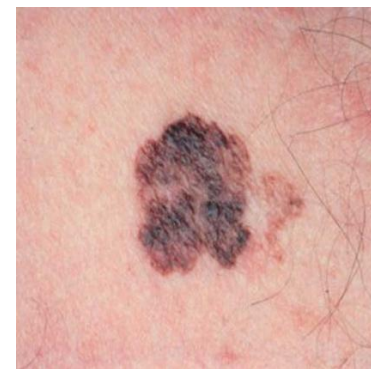

(a)

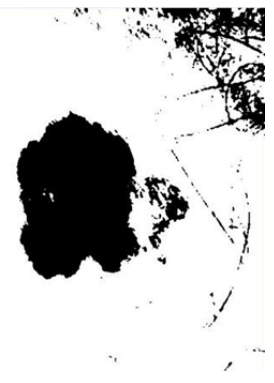

(b)
Fig. 4. (a) Input image and (b) OTSU processed image

$$
\begin{aligned}
& T=\text { weight } \times k \\
& k=\operatorname{Max}\left[\sqrt{w_{0}(k)\left(u_{0}(k)-u\right)^{2}+w_{1}(k)\left(u_{1}(k)-u\right)^{2}}\right]
\end{aligned}
$$

$\mathrm{w}_{0}$ and $\mathrm{w}_{1}$ denote the scales and $\mathrm{u}_{0}$ and $\mathrm{u}_{1}$ are the averages of the background and the foreground, respectively. $u$ is the average intensity of an image. In practical process, we make sure that only the normal skin part is in the foreground even if there are relatively few parts as shown in Fig. 4. After obtaining the normal skin area, the normal skin intensity value and their difference in each channel can be calculated.

Multichannel image processing is always timeconsuming especially with the increase in image size. After the input image is split into three single channels, we transform them into an integral image. By building the integral image, the sum of the pixel intensity in any rectangle region can be calculated by three times addition and subtraction. As a simple example, to sum up, the rectangular blocks with two corner points $v_{1}=\left(x_{1}, y_{1}\right)$ and $v_{2}=\left(x_{2}, y_{2}\right)$, then, $\sum_{x_{1} \leq x \leq x_{2}} \sum_{y_{1} \leq y \leq y_{2}}\left[I_{x, y}\right]$. Then, we defined the two components $x$ and $y$ to describe the skin pixel distribution by using the following logarithm;

$$
x_{i}=\log \frac{R_{i}}{G_{i}}, \quad y_{i}=\log \frac{B_{i}}{G_{i}}
$$

where $i$ is the index of a block of the integral image, RGB is the mean value. From the normal skin, the ratio $x_{0}$ and $y_{0}$ can be obtained as original values. We build vectors for the standard normal skin $v_{0}=\left(x_{0}, y_{0}\right)$, and the other skin area $v_{i}=\left(x_{i}, y_{i}\right)$. By deriving the functions, we can find a characteristic of the vectors, which is the normal skin vector $v_{i}=\left(x_{i}, y_{i}\right)$, is almost parallel to the standard vector $\mathrm{v}_{0}=\left(\mathrm{x}_{0}, \mathrm{y}_{0}\right)$. However, the pigment skin area is not parallel because the scale of RGB is changed. Therefore, the cosine similarity becomes a viable option to distinguish the pigment lesion and the normal area. Cosine similarity is the similarity measure of two vectors by measuring the cosine of the angle between them. Then, we can split the pigment area by using following equation.

$$
\operatorname{sim}=\cos (\theta)=\frac{V_{0} V_{1}}{\left\|V_{0}\right\|\left\|V_{1}\right\|}
$$

\subsection{Skin segmentation by Gaussian skin-color model}

Many studies presented methods for the detection and segmentation of skin areas [20-22]. Most of these methods extract features of skin-color pixels based on a variety of color space models. We tested the performance of skincolor clustering for various color space models and found that the $\mathrm{CbCr}$ components in the $\mathrm{YCbCr}$ color space produce the best clustering performance. The proposed scheme segments an input image into the skin and non-skin regions with a Gaussian skin color model in the $\mathrm{YCbCr}$ color space.

Given the $\mathrm{CbCr}$ components $X=\left(\boldsymbol{x}_{1}, \boldsymbol{x}_{2}, \cdots, \boldsymbol{x}_{N}\right)$; $\boldsymbol{x}_{j}=\left(C b_{j}, C r_{j}\right)$ of the $N$ pixels, the Gaussian skin-color model can be built from statistical information. We design the Gaussian skin-color model as $G(\boldsymbol{m}, \boldsymbol{C})$,

$$
\begin{gathered}
\boldsymbol{m}=(\overline{C b}, \overline{C r})^{T} \\
\boldsymbol{C}=E\left[(\boldsymbol{x}-\boldsymbol{m})(\boldsymbol{x}-\boldsymbol{m})^{T}\right]
\end{gathered}
$$

where $\boldsymbol{m}$ is the vector of the mean values of all pixels in the sample images and $\boldsymbol{C}$ is the covariance matrix. $\overline{C b}$ and $\overline{\mathrm{Cr}}$ are the means of $\mathrm{Cb}$ and $\mathrm{Cr}$ values. Following this approach, we calculate the values $G(\boldsymbol{m}, \boldsymbol{C})$ of the Gaussian skin-color model according to the statistical information from two hundred skin images that contain hands, arms, and faces in various illumination environments. We obtain an experience value as follows:

$$
\begin{gathered}
\boldsymbol{m}=(117.4361,156.5599) \\
\boldsymbol{C}=\left(\begin{array}{cc}
160.1301 & 12.1430 \\
12.1430 & 229.4574
\end{array}\right)
\end{gathered}
$$

We compute the skin similarity in terms of the Mahalanobis distance, $D(C b, C r)$. This means that the probability of a pixel belonging to a skin region depends on the distance from the center of a Gaussian distribution and is computed in terms of $D(C b, C r)$ as follows:

$$
\begin{aligned}
D(C b, C r) & =\sqrt{(\boldsymbol{x}-\boldsymbol{m})^{T} \boldsymbol{C}^{-1}(\boldsymbol{x}-\boldsymbol{m})} \\
\text { for } \quad \boldsymbol{x} & =(C b, C r)^{T}
\end{aligned}
$$

From the distance between the location of the input pixel and the center of the Gaussian skin-color model, we then determine whether a pixel has a skin color or a non-skin color on the basis of its threshold. When we use images from a facial image database as testing targets, the program has a very high rate of identification accuracy because of the simple content and great color difference with the background. However, in practice, there are always some pixels within an image that could be mistakenly identified due to their resemblance to skin color. When we set a very small threshold, the program will be too sensitive to the intensity changes of the skin, which results in several falsenegative pixels. On the other hand, when we set a very 
large threshold, the program may detect several falsepositive pixels. We opted for a smaller threshold in our program to avoid false-positive areas to be detected as pigmentation in subsequent processes or to influence the results. We selected the threshold by experimenting with statistical skin image values. We chose 30 practical images to test the skin detection program, and we attempted to control the threshold value to lose the least amount of skin area. Ultimately, the skin area missing to false negative pixels was $4 \%$ on the average. The Mahalanobis distance threshold of $D(C b, C r) \approx 0.790 .79$ is also obtained.

To improve accuracy, we tried using different threshold values for different kinds of skin, which improves the possibility of correctly detecting a skin area, but increases the false positive detections of the background at the same time.

\subsection{Hemoglobin-melanin decomposition}

ICA consists of a signal-processing algorithm that has been used since the 1990s. This method can discover the hidden factors or components from multi-dimensional statistical data. From the perspective of linear transformation and linear space, the source signals, which are independent non-Gaussian signals, can be classified as signals based on a linear space. The observed signal is a linear combination of the source signals. ICA employs an algorithm that can estimate the basic structure of the data space or source signal from an observed signal when the source signal or linear transformations are unknown.

This study focuses on the use of ICA as a feature extraction method. ICA algorithms search for underlying mutually independent components and extract the originally independent signals according to the analysis of statistical relationships among the observed multidimensional data. Feature extraction by ICA is mainly aimed at natural image processing. We have introduced the ICA algorithm to solve the problem of skin pigment detection. As mentioned previously, hemoglobin and melanin, along with their intensities, determine the color of the human skin. Hence, we use an algorithm to decompose the skin color into these two color components.

The ICA algorithm can extract the original signals from mixtures of many independent sources without a priori information on the sources and processes of the mixture. ICA finds independent skin color components by maximizing the statistical independence of the estimated components. A neural network algorithm can perform ICA calculations. Independence can also be defined, which governs the form of the ICA algorithm. For a human skincolor model, the ICA is based on three assumptions: 1) the spatial variation of skin color is caused by two components, which are hemoglobin and melanin; 2) the quantities of these components are independent of each other, and 3) the linearity holds among the quantities and the observed color signals in the optical density domain.

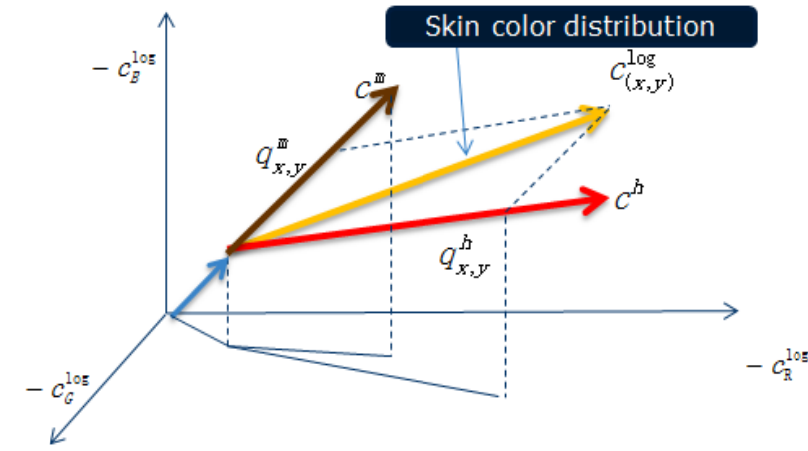

Fig. 5. Density vectors of hemoglobin and melanin using ICA algorithms

Tsumura et al. [23, 24] extracted the hemoglobin and melanin images from a human skin-color image using an ICA algorithm. Based on the linearity assumption, the skin-color model in the optical density domain of the RGB channels can be expressed with the color density vector $\boldsymbol{L}_{x, y}$.

$$
\boldsymbol{L}_{x, y}=\left[\log \left(\frac{1}{r_{x, y}}\right), \log \left(\frac{1}{g_{x, y}}\right), \log \left(\frac{1}{b_{x, y}}\right)\right]^{T}
$$

Let $\mathrm{T}$ represent the transposition of a matrix. If $r_{x, y}$, $g_{x, y}$, and $b_{x, y}$ are pixels of the R, G, and B channels, respectively, then $\boldsymbol{L}_{x, y}$ can be rewritten by using the hemoglobin component $m_{x, y}$ and melanin component $h_{x, y}$, as shown in Fig. 5.

$$
\boldsymbol{L}_{x, y}=\boldsymbol{C}^{T}\left[m_{x, y}, h_{x, y}\right]+\Delta \text { where } \boldsymbol{C}=\left[\boldsymbol{c}^{m}, \boldsymbol{c}^{h}\right]
$$

Note that $\boldsymbol{c}^{m}$ and $\boldsymbol{c}^{h}$ are the pure density vectors of melanin and hemoglobin while $m_{x, y}$ and $h_{x, y}$ are the relative hemoglobin and melanin quantities of these pigments. The spatially stationary vector $\Delta$ is caused by other skin structures. Principal component analysis (PCA) is used to extract into a $2 \mathrm{D}$ plane. The independence evaluation of Burel [25] can be used for measuring the independence of a component. We obtain the minimizing values for the elements of a vector by this evaluation. This minimization process is performed by a quasi-Newtonian implementation using the MATLAB toolbox. We apply an ICA to estimate the relative quantities. Thus, the skin color can be decomposed as follows:

$$
\boldsymbol{L}_{x, y}^{\prime}=\widetilde{\boldsymbol{C}}\left(\boldsymbol{K}\left[m_{x, y}, h_{x, y}\right]^{T}+j \boldsymbol{E}\right)+j \Delta
$$

where $\left[m_{x, y}, h_{x, y}\right]=\widetilde{\boldsymbol{C}}^{-1} \boldsymbol{L}_{x, y}-\boldsymbol{E}$

$$
E=\min _{x, y}\left(\widetilde{\boldsymbol{C}}^{-1} \boldsymbol{L}_{x, y}\right)
$$

$\boldsymbol{L}_{x, y}^{\prime}$ is the synthesized skin color, and $\widetilde{\boldsymbol{C}}$ is the estimate $\left[m_{x, y}, h_{x, y}\right]$, while $\boldsymbol{K}$ and $j$ are synthesis parameters. To obtain the melanin and hemoglobin components for a skin region, we set two values of the synthesis parameter $\boldsymbol{K}$ : 
$\boldsymbol{K}=\operatorname{diag}[1,0]$ and $\boldsymbol{K}=\operatorname{diag}[0,1]$.

Through the above process, the color of a skin region with $N V \times N H$ size is decomposed into hemoglobin component $\boldsymbol{M}=\left\{m_{x, y} \mid x \in[1, N H], y \in[1, N V]\right\} \quad$ and melanin component $\boldsymbol{H}=\left\{h_{x, y} \mid x \in[1, N H], y \in[1, N V]\right\}$. From the tested images, the intensity of melanin components $\boldsymbol{M}$ is close to uniform and the intensity of hemoglobin components $\boldsymbol{H}$ is low.

\subsection{Hemoglobin-melanin location histogram}

An HM location histogram reflects the spatial distribution of hemoglobin $\mathrm{H}$ and melanin $\mathrm{M}$ components. The location histogram has three axes denoted as $\mathrm{x}, \mathrm{y}$, and $\mathrm{z}$. The z-axis defines the intensity of a pixel while the $\mathrm{x}$ - and $y$-axes define the spatial location of a pixel within an image. The $N V \times N H$ size of $\boldsymbol{H}$ and $\boldsymbol{M}$ is divided into rectangular bins $\boldsymbol{B}^{H}$ and $\boldsymbol{B}^{M}$ with $\Delta x \times \Delta y$.

$$
\begin{aligned}
\boldsymbol{B}^{H}=\left\{B_{i j}^{H}\right. & =\left\{h_{x, y} \mid x \in[(i-1) \Delta x, i \Delta x],\right. \\
y & \in[(j-1) \Delta y, j \Delta y]\}\} \\
\boldsymbol{B}^{M}=\left\{B_{i j}^{M}\right. & =\left\{m_{x, y} \mid x \in[(i-1) \Delta x, i \Delta x],\right. \\
y & \in[(j-1) \Delta y, j \Delta y]\}\}
\end{aligned}
$$

for all $i \in\left[0, N_{H}-1\right], j \in\left[0, N_{V}-1\right]$

The magnitude of each bin is the mean intensity of the components in a corresponding bin.

$$
\begin{aligned}
b_{i j}^{M} & =\frac{1}{\Delta x \times \Delta y} \int_{(i-1) \Delta x}^{i \Delta x} \int_{(j-1) \Delta y}^{j \Delta y} m_{x, y} d y d x \\
b_{i j}^{H} & =\frac{1}{\Delta x \times \Delta y} \int_{(i-1) \Delta x}^{i \Delta x} \int_{(j-1) \Delta y}^{j \Delta y} h_{x, y} d y d x
\end{aligned}
$$

The number of bins $N_{H} \times N_{V}$ affects the intensity distribution of hemoglobin and melanin, which depends on the bin size $\Delta x \times \Delta y$.

$$
N_{H}=\left\lceil\frac{N H}{\Delta x}\right\rceil \text { and } N_{V}=\left\lceil\frac{N V}{\Delta y}\right\rceil
$$

Generally, the normal skin has a higher intensity than its surroundings, but skin pigment has a low intensity. These properties can be used for detecting skin pigment. The number of bins should be used to control the accuracy performance. Therefore, we determine the bin size $\Delta x$ and $\Delta y$ by the minimization of an estimated $L^{2}$ risk function [26].

$$
(\Delta x, \Delta y)=\left(\arg \min _{\Delta x} \frac{2 \mu_{x}-\sigma_{x}}{\Delta x^{2}}, \arg \min _{\Delta y} \frac{2 \mu_{y}-\sigma_{y}}{\Delta y^{2}}\right)
$$

$\left(\mu_{x}, \mu_{y}\right)$ and $\left(\sigma_{x}, \sigma_{y}\right)$ are the mean and biased variance of bin magnitude of hemoglobin and melanin on each coordinate.

$$
\left(\mu_{x}, \mu_{y}\right)=\left(\frac{1}{N_{H}} \sum_{i=0}^{N_{H}-1}\left(\frac{b_{i}^{H}+b_{i}^{M}}{2}\right), \frac{1}{N_{H}} \sum_{j=0}^{N_{V}-1}\left(\frac{b_{j}^{H}+b_{j}^{M}}{2}\right)\right)
$$

$$
\begin{aligned}
& \left(\sigma_{x}, \sigma_{y}\right)= \\
& \left(\frac{1}{N_{H}} \sum_{i=0}^{N_{H}-1}\left(\frac{b_{i}^{H}+b_{i}^{M}}{2}-\mu_{x}\right)^{2}, \frac{1}{N_{H}} \sum_{j=0}^{N_{V}-1}\left(\frac{b_{j}^{H}+b_{j}^{M}}{2}-\mu_{y}\right)^{2}\right.
\end{aligned}
$$

where $\left(b_{i}^{H}, b_{j}^{H}\right)$ and $\left(b_{i}^{M}, b_{j}^{M}\right)$ are 1D mean integral of hemoglobin and melanin components on each coordinate.

$$
\begin{aligned}
& \left(b_{i}^{H}, b_{j}^{H}\right)=\left(\frac{1}{\Delta x} \int_{(i-1) \Delta x}^{i \Delta x} h_{x, y} d x, \frac{1}{\Delta y} \int_{(j-1) \Delta y}^{j \Delta y} h_{x, y} d y\right) \\
& \left(b_{i}^{M}, b_{j}^{M}\right)=\left(\frac{1}{\Delta x} \int_{(i-1) \Delta x}^{i \Delta x} m_{x, y} d x, \frac{1}{\Delta y} \int_{(j-1) \Delta y}^{j \Delta y} m_{x, y} d y\right)
\end{aligned}
$$

The location histograms on HM projective coordinates are shown in Fig. 6, where a skin-segmented image is divided into bins within the square of bin numbers.

\subsection{Pigment labeling}

Given that the face or body is not flat, the intensity is never uniform even under constant illumination, as shown in Fig. 7(a). Regardless of shadow, the pigment must be detected in all regions of the skin. To minimize the influence of shadow, the local distributions of hemoglobin and melanin should be considered. Thus, to classify three types of hemoglobin, melanin, and normal, we use the

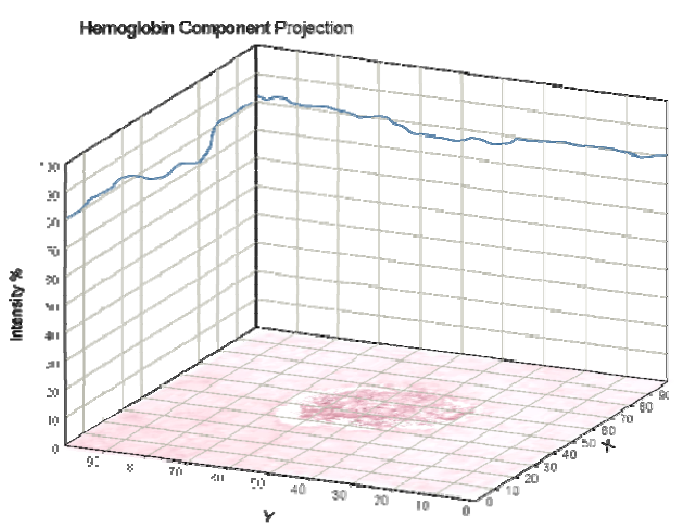

(a)

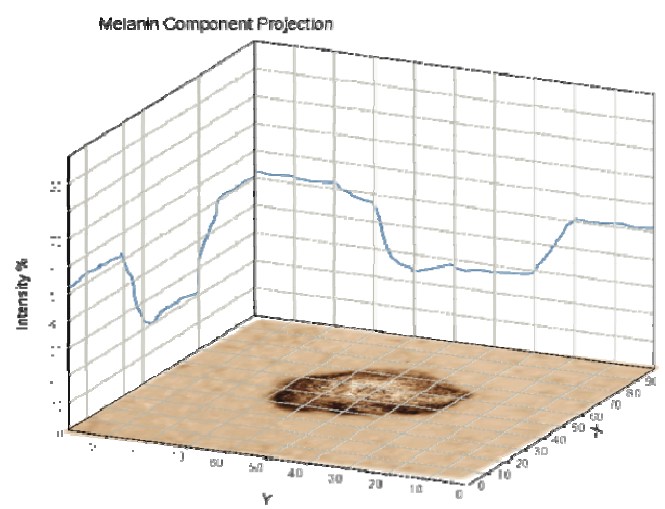

(b)

Fig. 6. 2D location histograms for (a) hemoglobin and (b) melanin coordinates 

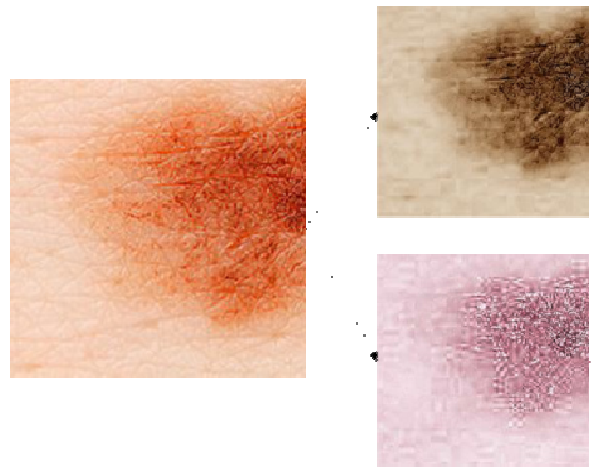

(a)
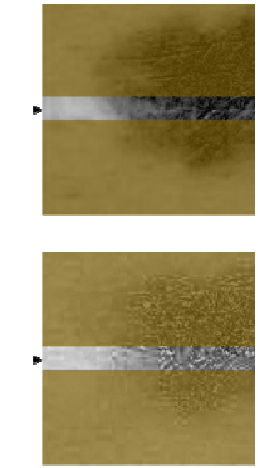

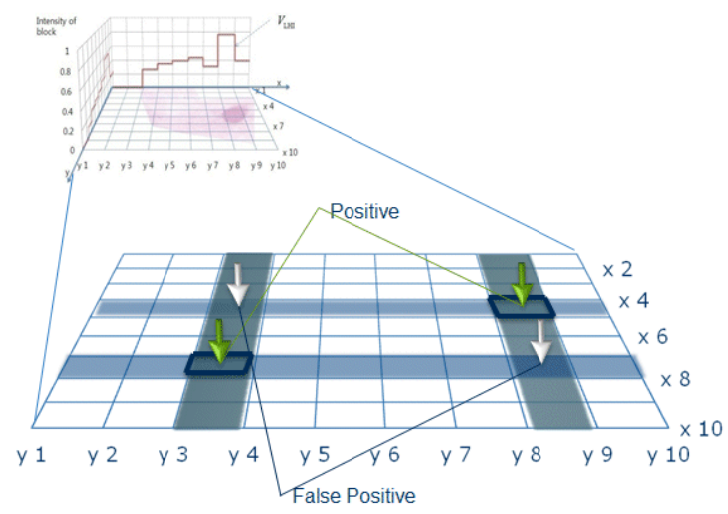

(b)

Fig. 7. (a) Non-uniform intensity on a skin by the unevenness of skin surface and (b) possibility of false positive

local ratio $\mathrm{L}$ of hemoglobin and melanin components. The local ratio $L_{i j}$ is defined by the ratio of hemoglobin and melanin components on a bin.

$$
\begin{aligned}
L_{i j}= & \frac{b_{i j}^{H}}{b_{i j}^{M}}=\frac{\frac{1}{\Delta x \times \Delta y} \int_{(i-1) \Delta x}^{i \Delta x} \int_{(j-1) \Delta y}^{j \Delta y} m_{x, y} d y d x}{\frac{1}{\Delta x \times \Delta y} \int_{(i-1) \Delta x}^{i \Delta x} \int_{(j-1) \Delta y}^{j \Delta y} h_{x, y} d y d x} \\
& \approx \int_{(i-1) \Delta x}^{i \Delta x} \int_{(j-1) \Delta y}^{j \Delta y} \frac{h_{x, y}}{m_{x, y}} d y d x
\end{aligned}
$$

Finally, the presence of hemoglobin and melanin in a bin is determined by comparing the local ratio $L_{i j}$ to the reference $R$, as shown in Fig. 8.

$$
B_{i j}=\left(\begin{array}{cc}
\text { Hemoglobin, } & \text { if } L_{i j}>R+\varepsilon \\
\text { Normal skin, } & \text { if } R-\frac{1}{\varepsilon} \leq L_{i j} \leq R+\varepsilon \\
\text { Melanin, } & \text { if } L_{i j}<R-\frac{1}{\varepsilon}
\end{array}\right.
$$

If the local ratio $L_{i j}$ approaches to 1 , which means that both hemoglobin and melanin are distributed equally, this bin is the normal skin. The reference $\mathrm{R}$ is assumed to be 1 with constant on all cases. However, to consider adaptively illuminations, we set the reference to the global ratio of hemoglobin and melanin on a skin segmented image.

$$
\begin{aligned}
R=\frac{\bar{H}}{\bar{M}} & =\frac{\frac{1}{N V \times N H} \int_{0}^{N H} \int_{0}^{N V} h_{x, y} d y d x}{\frac{1}{N V \times N H} \int_{0}^{N H} \int_{0}^{N V} m_{x, y} d y d x} \\
& \approx \int_{0}^{N H} \int_{0}^{N V} \frac{h_{x, y}}{m_{x, y}} d y d x
\end{aligned}
$$

Our proposed method can detect pigmentation and recalculate the region of interest in the skin by using the location histogram. Furthermore, the 2D parameters allowed faster processing. However, if the edge detection is not exact or there is any skin pigment region within an image, the blocks might be falsely labeled as shown in Fig. 7(b). To solve this problem, we use the same ratio to recalculate the regions of interest showing pigment that were labeled in the previous step. Thus, the regions of

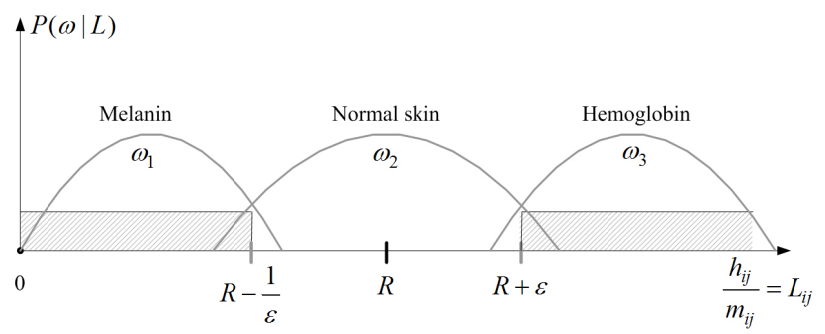

Fig. 8. Classification of normal skin and two pigments of melanin and hemoglobin

interest showing pigment are recalculated as a unit (or block) to remove false positive labels.

We assume that for a given illumination environment, a local area of the skin has almost the same intensity as the global area. Hence, only the pigmentation element can influence the ratio $L$. We compared the hemoglobin and melanin components of 30 pigment images. We found that if the intensity difference between a pigmented area and its surrounding normal skin approaches 35 , a slight difference will be observed. If the intensity difference reaches 60 , a conspicuous difference will be observed. Skin pigment is distinguished as shown in Fig. 7(a). We set the threshold $\varepsilon$ according to the general difference between pigment regions and normal skin in sample images.

We can classify three types of normal skin and two pigments of hemoglobin and melanin using Bayesian classification, which we denote three classes by $\omega_{1}, \omega_{2}$, and $\omega_{3}$. Given the local ratio vectors $\boldsymbol{L}=\left\{L_{i j}\right\}$, we determine the threshold $\varepsilon$ that minimizes the expected error rate for the three classes and unequal priors.

$$
\arg \min _{\varepsilon}\left[P\left(\omega_{i} \mid L\right)>P\left(\omega_{j} \mid L\right) \mid \forall j \neq i \rightarrow \omega_{i}\right]
$$

The prior probabilities of the three classes are $P\left(\omega_{1}\right)$, $P\left(\omega_{2}\right)$, and $P\left(\omega_{3}\right)$. The likelihoods are $P\left(L \mid \omega_{i}\right)$ for $i=1,2,3$. The posteriors in the above equation are

$$
P\left(\omega_{i} \mid L\right)=\frac{P\left(L \mid \omega_{i}\right) P\left(\omega_{i}\right)}{P(L)}
$$



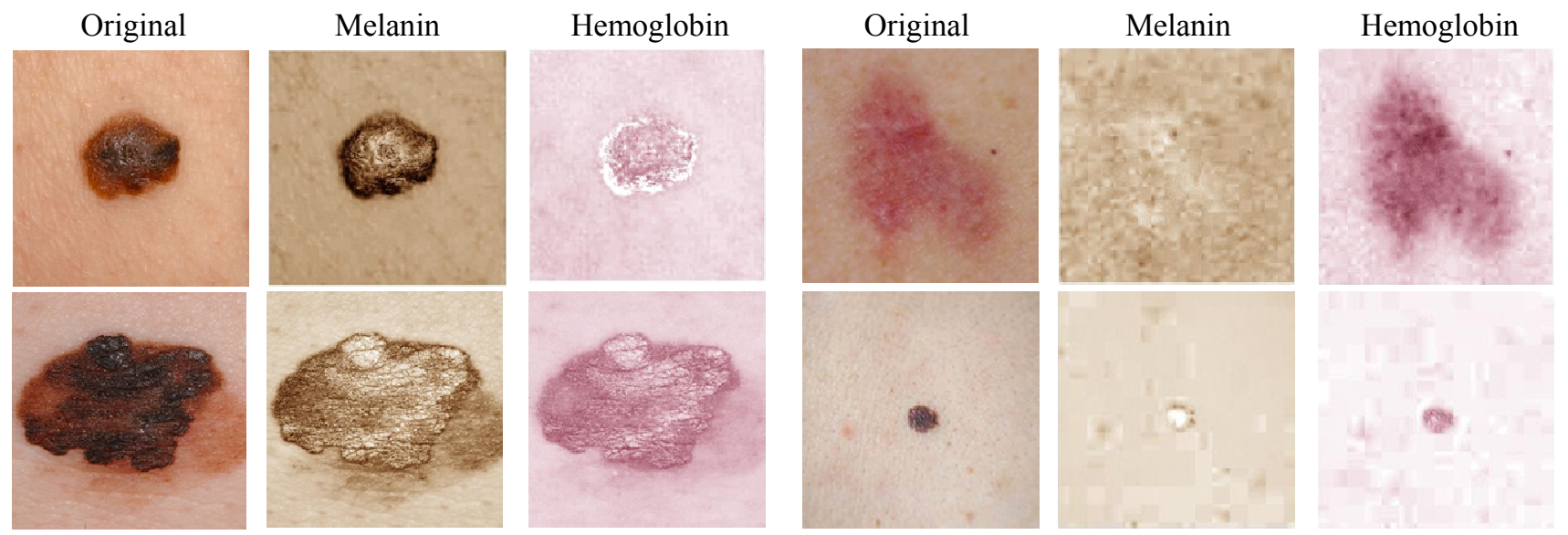

Fig. 9. Pigmented sample images: (a) First column shows skin with black spot or skin with a red spot; (b) the second column is the melanin component, and (c) the third column is the hemoglobin component of the skin

where $P(L)=\sum_{i=1}^{3} P\left(L \mid \omega_{i}\right) P\left(\omega_{i}\right)$.

\subsection{Pigment quantification}

We measure the quantity of skin pigment using the location histogram. The area of skin pigment can be calculated from the percentage of positive blocks. When the number of bins is larger, the accuracy will be higher. The area $A(p)$ will be such that $p$ is the number of positive pigment blocks.

$$
A(p)=\frac{p}{\left(N_{H} \times N_{V}\right)^{2}} \times N V \times N H
$$

Additionally, $N_{H} \times N_{V}$ is the number of histogram bins, and $(N V, N H)$ denotes the size of the segmented skin image.

The degree of skin pigment can be obtained from the standard deviation $\sigma$ that indicates the difference in the intensity between the pigmented skin and the surrounding normal skin.

$$
\sigma=\sqrt{\frac{1}{N} \sum_{i=1}^{N}\left(x_{i}-\bar{x}\right)^{2}}
$$

Note that $x$ is the pixel intensity, $\bar{x}$ is the average intensity of the test images, and $N$ is the number of pixels. Through the area percentage and intensity deviation of the skin pigment, we can digitize the pigment for measurement. Then, we can distinguish which measure is more accurate across many samples. Furthermore, our scheme can be applied to measure the variation in a skin condition before and after makeup or treatment. D. Zhang et al. proposed the de-noising method using the spherical coordinate system that is re-defined in the wavelet domain [27-30]. If the de-noising method can be applied to the first step of our method, we think that the standard deviation of our method can be computed with more accuracy.

\section{Experimental Results}

\subsection{Experiment setting}

Our tested images were taken under typical real-life conditions. We used a green mask to label the skin pigment and output two measures, namely, the area of the skin pigment and the standard deviation. Our proposed method consists of three steps, namely, skin area segmentation, decomposition of skin color into hemoglobin and melanin components, and detection of skin pigment based on the location histogram. In our experiments, we segmented a number of skin images from 200 natural images with a face, arm, and hand for the first step. The second step processed the color decomposition or the skin region based on an ICA algorithm. As expected, the color of a skin region can be well decomposed into its hemoglobin component and melanin component, as shown in Fig. 9, where hemoglobin is colored in black-pigmented spots and melanin is colored in red-pigmented spots from a number of skin types. The figures show that our method performs well in identifying hemoglobin and melanin components in skin regions. We retained the influence of pigmentation only relative to its own component. Then, we can measure the intensity distribution in the next step of our method.

We calculated the location histogram along the $\mathrm{x}$ - and $\mathrm{y}$ axes of the hemoglobin and melanin components. Then, the matching bin values are compared to the global and local ratios. The experiment shows that the local ratio for a normal skin area is close to the global ratio for an image. However, if some bins include red or black spots, the local ratio will be further away from the global value. Previously, we used a threshold to detect the blocks containing pigmentation, namely, 30 and 100, which represent the two bins used for testing. When we abandon absolute precision, we can acquire a significantly increased processing speed.

We compared our scheme with that of $\mathrm{Lu}$ [12], which 
segments the skin pigment from different images, but does not quantify the skin pigments. They detected the skin region with a histogram-based Bayesian classifier and extracted the pigments of melanin and hemoglobin based on an ICA algorithm. Then, they identified the erythemic regions by using a support vector machine (SVM) for two components. This method can detect well in low-contrast

Table 1. Recognition results on various illuminations of our method.

\begin{tabular}{l|c|c|c}
\hline \multicolumn{1}{c|}{ Illuminations } & Area rate & MSE (Pigment degree) & Error rate \\
\hline 1) Dark Shadow & $5.93 \%$ & 16.02 & $21.83 \%$ \\
\hline 2) Medium Shadow & $10.50 \%$ & 17.05 & $51.77 \%$ \\
\hline 3) Light Shadow & $6.52 \%$ & 14.52 & $1.26 \%$ \\
\hline 4) Lights & $8.66 \%$ & 19.05 & $0 \%$ \\
\hline 5) Sun & $7.14 \%$ & 15.59 & $1.17 \%$ \\
\hline 6) Specular & $3.97 \%$ & 7.52 & $47.71 \%$ \\
\hline
\end{tabular}
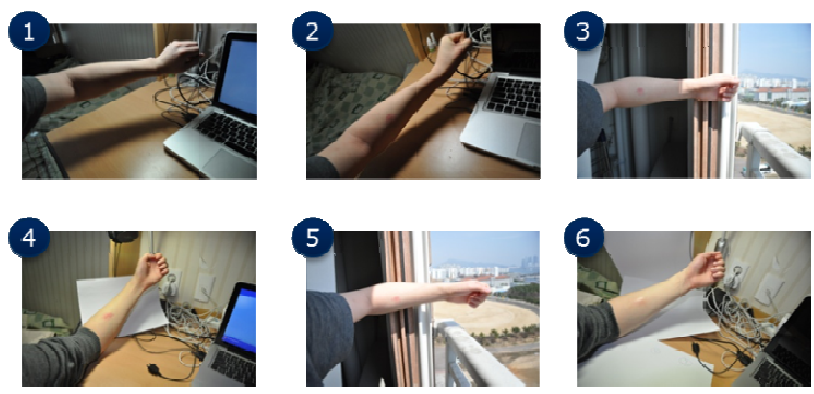

Fig. 10. Experimental conditions of the tested illumination; 1) Dark shadow, 2) medium shadow, 3) light shadow, 4) lights, 5) sun, and 6) specular
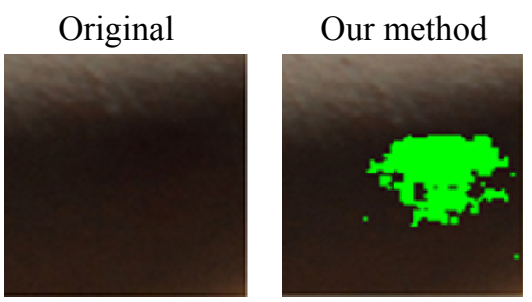

(a)
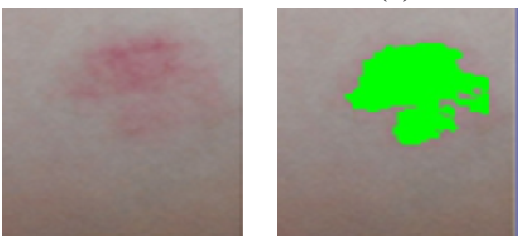

(c)
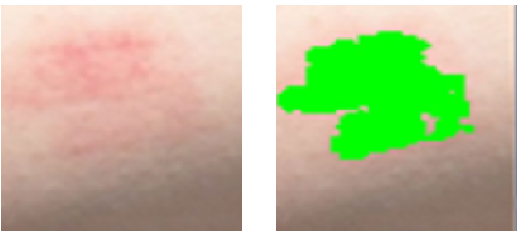

(e)
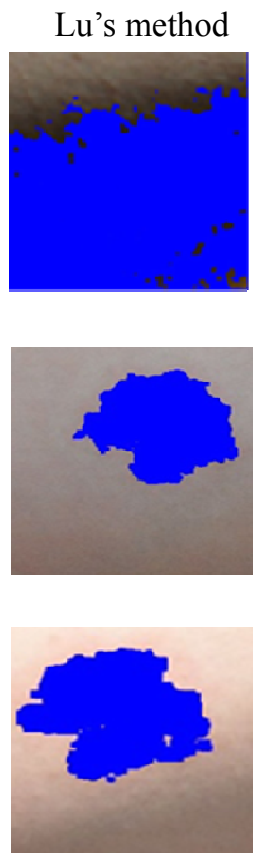

images, but needs long processing time and has limited adaptability under different illumination conditions.

\subsection{Recognition performance on illuminations}

The recognition performance of our proposed method is tested on six different illumination conditions as shown in Fig. 10. Thus, 100 test images with single and multiple light sources and sunlight, including dark shadow, medium shadow, light shadow, lights, sun, and specific, were used. These test images were captured from a Nikon D90 camera on AWB (automatic white balance). The detected results of our method are shown in Fig. 11, where the skin pigments are colored to green mask. We output two quantities, namely, the area percentage and the standard deviation of skin pigments. Table 1 shows the two output quantities of the tested images. The figures and tables show that our method has the adaptability to certain light conditions and has very low error rate on dark shadow and specific illuminations.

Fig. 12(a) shows the recognition rate of pigments of our proposed method and Lu's method, which are average recognition rate of all test images on six kinds of illuminations. The pigment detection should consider both the false detection and the illumination effect to identify hemoglobin and melanin. False detection denotes false identification of a non-skin to skin. Our proposed method reduced the illumination effect by comparing the local ratio and the global ratio of $\mathrm{HM}$ projective histograms. Our method and Lu's method recognized well all pigments in medium brightness. However, both methods failed to
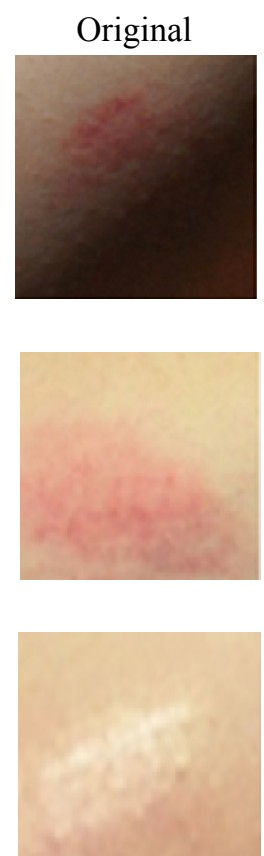

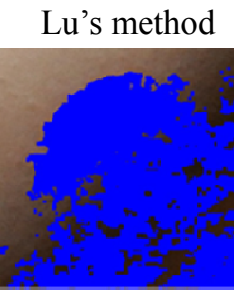

(b)

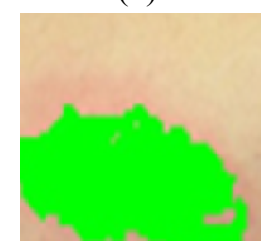

(d)

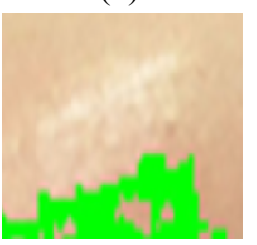

(f)
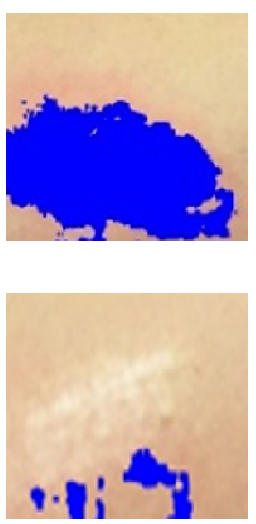

Fig. 11. Recognized results of our method and Lu's method on various illuminations of (a) Dark Shadow, (b) Medium shadow, (c) Light shadow, (d) Direct light, (e) Sun, and (f) Specular 


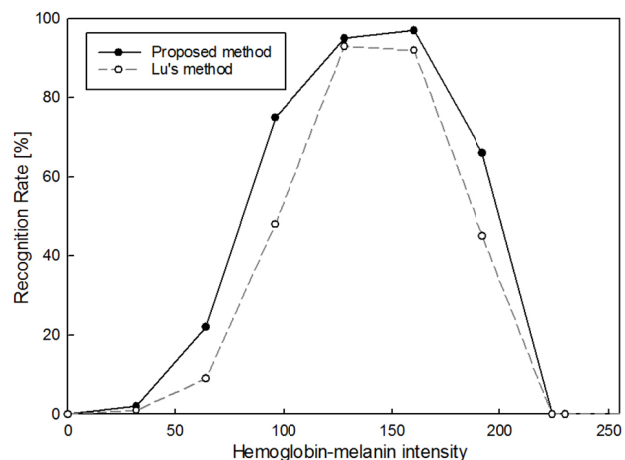

(a)

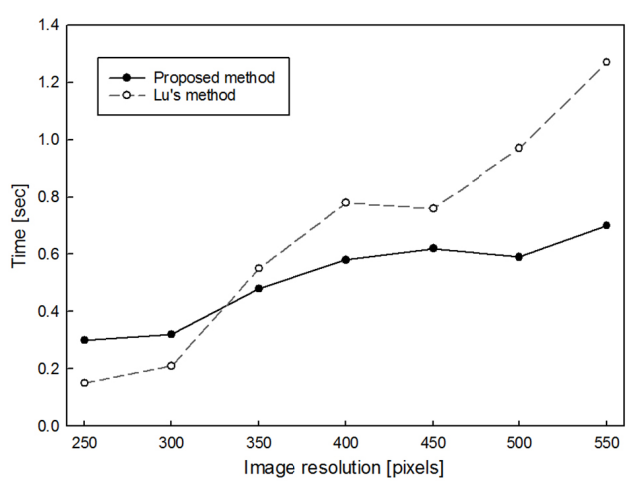

(b)

Fig. 12. (a) Pigment recognition rate and (b) processing time of our method and Lu's method

recognize any pigments on very low and high brightness conditions. Thus, in dark shadow and high brightness, our proposed method has $25 \%$ more recognition rate than Lu's method.

\subsection{Processing Time Performance}

For the process time test, the computer configuration used is as follows: CPU-Core2 $2.8 \mathrm{GHz}$, RAM-3GB, OSWinXP, Graphics card-ATI HD3400. We find that the image content barely affects the process time; therefore, for each size level, we only tested three images and compared the average value. In small cases, both approaches can quickly finish the process. However, when the image size is increased, the performance of our approach is obviously better than that of Lu's method as shown in Fig. 12(b). The process of skin segmentation and pigment detection is similar to an image classification using three color channels. This process requires longer time and larger memory to be computed. Our method uses only two channels of $\mathrm{Cb}, \mathrm{Cr}$ that reduces the computation time and memory. Furthermore, our method decomposes the skin into two channels of hemoglobin and melanin by ICA algorithm. Given these reasons, our method has shorter processing time than Lu's method as shown in Fig. 12(b). Our method can quantify the density of skin pigments using the HM projective location histograms; therefore, it
Table 2. Results of cosmetic covering effect by our method and Lu's method (light illumination)

\begin{tabular}{|c|c|c|c|c|}
\hline \multirow[b]{2}{*}{ Sample } & \multicolumn{3}{|c|}{ Our method } & \multirow{2}{*}{$\begin{array}{l}\text { Lu's } \\
\text { method } \\
\text { error } \\
\text { rate } \\
\end{array}$} \\
\hline & $\begin{array}{c}\text { Detected } \\
\text { area }\end{array}$ & $\begin{array}{c}\text { Standard } \\
\text { deviation } \sigma \\
\text { of pigments }\end{array}$ & $\begin{array}{l}\text { Error } \\
\text { rate }\end{array}$ & \\
\hline Skin with no pigments & $0.00 \%$ & - & - & - \\
\hline Skin with pigments & $8.53 \%$ & 18.73 & $0 \%$ & $2.5 \%$ \\
\hline $\begin{array}{c}\text { Cosmetic Sample1 } \\
\text { (Age away primer base) }\end{array}$ & $0.11 \%$ & 8.85 & $6.8 \%$ & $10.4 \%$ \\
\hline $\begin{array}{c}\text { Cosmetic Sample2 } \\
\text { (blemish cover balm) }\end{array}$ & $0.02 \%$ & 7.35 & $10.5 \%$ & $15.2 \%$ \\
\hline $\begin{array}{l}\text { Cosmetic Sample3 } \\
\text { (BB magic cream) }\end{array}$ & $0.13 \%$ & 8.13 & $5.5 \%$ & $8.9 \%$ \\
\hline
\end{tabular}

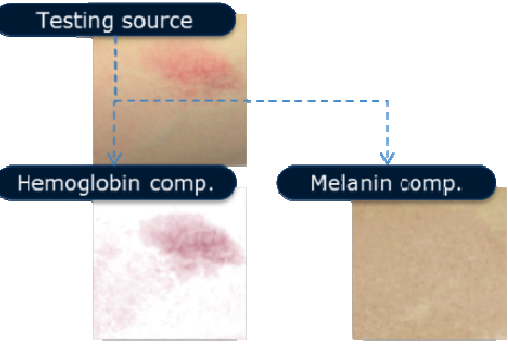

(a)

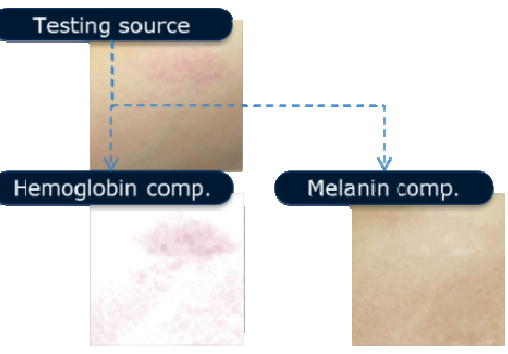

(c)

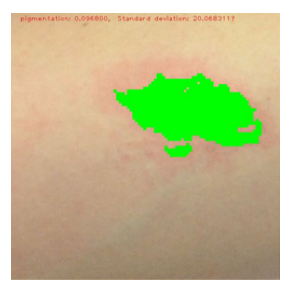

(b)

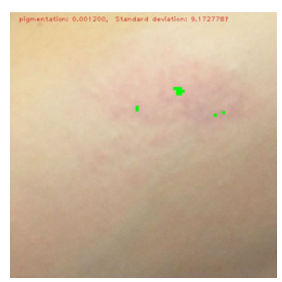

(d)
Fig. 13. (a) Skin with pigments without cosmetic and (b) detected pigments; (c) Covered skin with pigments with Cosmetic Sample 2 (blemish cover balm) and (d) detected pigments in the covered skin

has shorter processing time and is more intuitive to the skin description.

\subsection{Cosmetic covering evaluation}

To attractive applications, our method can evaluate the effect of cosmetic covering on an abnormal skin. In our cosmetic evaluation experiments, we selected three brands of cosmetic products with the function that covers the skin pigments. We placed $0.05 \mathrm{ml}$ of cosmetic on skin pigments. Then, we measured the two quantities of skin pigments with or without cosmetic. The selected cosmetics are: 1) age away primer base, 2) blemish cover balm, 3) BB magic cream. The detection results of skin pigments on cosmetic covering are described in Table 2. Especially, the detected 
pigments for skin image without cosmetic and with cosmetic sample 2 are shown in Fig. 13. The reference sample of the skin pigment without the cosmetic is detected to about $8.54 \%$ pigmented area and 18.3 standard deviation $\sigma$. Cosmetic Sample 1 and Sample 3 reduce the detected area to about $0.11 \%-0.13 \%$. The reduction of cosmetic Sample 2 to about $0.02 \%$ denotes that it covers fully the skin pigment. Although the detected area and deviation are reduced to high percentage, our method detected the presence of skin pigments at above $80 \%$. However, Lu's method failed to detect about 2.5\%-4.7\% more than our method.

\section{Conclusion}

This study addressed the recognition and measurement of the human skin pigment using hemoglobin-melanin projective coordinate. We used skin-color clustering in the YCbCr color model to build a Gaussian skin-color model. Then, this model is used to segment the skin area from the background of the image. Next, we decomposed the skin image into hemoglobin and melanin images using an ICA algorithm. We included a location histogram to calculate the intensities of pixels. From the intensity values, we computed the global and local ratios, which we compared to determine the occurrence of pigmentation. Moreover, we found some minor processing steps of the program that require improvement to for better performance. In our testing phase, the program performed with a good processing time and detection rate in most cases. However, for special cases, such as dark shadow, the program also revealed a weakness that needs to be rectified. In future work, we consider using different ICA estimation algorithms to test for performance and stability. Finally, the experimental result supports our scheme as an effective means of human skin pigment detection.

\section{Acknowledgements}

This research was supported by the ICT R\&D program of MSIP/IITP [R0126-15-1112, Development of Media Application Framework based on Multi-modality which enables Personal Media Reconstruction] and also by Basic Science Research Program through the National Research Foundation of Korea(NRF) funded by the Ministry of Education, Science and Technology(NRF-2011-0023118 and NRF-2014R1A1A4A01006663) and also the MSIP (Ministry of Science, ICT and Future Planning), Korea, under the Grand Information Technology Research Center support program (IITP-2016-R71181610050001002) supervised by the IITP(Institute for Information \& communications Technology Promotion).

\section{References}

[1] G. Sforza, G. Castellano, R. J. Stanley, W. V. Stoecker, and J. Hagerty, "Adaptive segmentation of gray areas in dermoscopy images," Medical Measurements and Applications Proceedings (MeMeA), pp. 628-631, May. 2011.

[2] O. Sarrafzade, M. H. M. Baygi, and P. Ghassemi, "Skin lesion detection in dermoscopy images using wavelet transform and morphology operations," Biomedical Engineering (ICBME), pp. 1-4, Nov. 2010.

[3] H. Zhou, J.M. Rehg, and M. Chen, "Exemplar-based segmentation of pigmented skin lesions from dermoscopy images," Biomedical Imaging: From Nano to Macro, pp. 225-228. Apr. 2010.

[4] Y. Liu, S.-H. Lee, S.-G. Kwon, and K.-R. Kwon, "Skin-pigmentation-disorder detection algorithm based on projective coordinate," Optik, vol. 127, issue 15, pp. 5899-5913, Aug. 2016.

[5] A. Krishnaswamy and G. V. G Baranoski, A study on skin optics, Canada: University of Waterloo, 2004.

[6] H. Nugroho, A. F. M. Hani, R. Jolivot, and F. Marzani, "Melanin type and concentration determination using inverse model," National Postgraduate Conference (NPC), pp. 1-7. Sep. 2011.

[7] V. K. Madasu, and B. C. Lovell, "Blotch detection in pigmented skin lesions using fuzzy co-clustering and texture segmentation," Digital Image Computing: Techniques and Applications, pp. 25-31, Dec. 2009.

[8] K. M. Clawson, P. J. Morrow, B. W. Scotney, D. J. Mckenna, and O. M. Dolan, "Computerised skin lesion surface analysis for pigment asymmetry quantification," Machine Vision and Image Processing Conference, pp. 75-82, Sep. 2007.

[9] W. R. Tan, C. S. Chan, P. Yogarajah, and J. Condell, "A Fusion Approach for Efficient Human Skin Detection," IEEE Transactions on Industrial Informatics, vol. 8, no. 1, pp. 138-147, Feb. 2012.

[10] M. Shoyaib, M. Abdullah-Al-Wadud, O. Chae, and R. Byungyong, "Skin detection using statistics of small amount of training data," Electronics Letters, vol. 48, no. 2, pp. 87-88, Jan. 2012.

[11] L. Liu, N. Sang, S. Yang, and R. Huang, "Real-time skin color detection under rapidly changing illumination conditions," IEEE Transactions on Consumer Electronics, vol. 57, no. 3, pp. 1295-1302, Aug. 2011.

[12] J. Lu, J. H. Manton, E. Kazmierczak, and R. Sinclair, "Erythema detection in digital skin images," Image Processing (ICIP), pp. 2545-2548. Sep. 2010.

[13] Z. F. Khan and A. Kannan, "Intelligent approach for segmenting CT lung images using fuzzy logic with bitplane," Journal of Electrical Engineering and Technology, vol. 9, no. 4, pp. 742-752, 2014.

[14] Z. F. Khan and A. Kannan, "Intelligent segmentation of medical images using fuzzy bitplane thresholding," Measurement Science and Review, vol. 14, no. 2, pp. 
94-101, 2014.

[15] Z. F. Khan and S. U. Quadri, "FEMD algorithm for effective segmentation of CT lung images," International Journal of Computer Applications, vol. 111, no. 8, pp. 21-24, Feb. 2015.

[16] M. Fotouhi, M. H. Rohban, and S. Kasaei, "Skin detection using contourlet-based texture analysis," Fourth International Conference on Digital Telecommunications, ICDT, pp. 59-64. Jul. 2009.

[17] Zh. M. Li, T. Zhang, and J. Zhang, "Skin detection in color images," Computer Engineering and Technology (ICCET), vol. 1, pp. 156-159. Apr. 2010.

[18] R. L. Hsu, M. Abdel-Mottaleb, and A. K. Jain, "Face detection in color images," Pattern Analysis and Machine Intelligence, vol. 24, no. 5, pp. 696-706. 2002.

[19] S. Kherchaoui, and A. Houacine, "Face detection based on a model of the skin color with constraints and template matching," International Conference on Machine and Web Intelligence (ICMWI), pp. 469-472. Oct. 2010.

[20] S. L. Phung, D. Chai, and A. Bouzerdoum, "Adaptive skin segmentation in color images," IEEE International Conference on Acoustics, Speech, and Signal Processing, vol. 3, pp. 353-356, Apr. 2003.

[21] S. L. Phung, A. Bouzerdoum, and D. Chai, "Skin segmentation using color pixel classification: analysis and comparison," IEEE Transactions on Pattern Analysis and Machine Intelligence, vol. 27, pp. 148154, Jan. 2005.

[22] R. Hassanpour, A. Shahbahrami, and S. Wong, "Adaptive Gaussian mixture model for skin color segmentation," Proceedings of World academy of Science, Engineering and Technology, vol. 31, pp. 16, July 2008.

[23] N. Tsumura, H. Haneishi, and Y. Miyake, "Independent component analysis of skin color model image," Journal of Optical Society of America A, vol. 16, no. 9, pp. 2169-2176, 1999.

[24] N. Tsumura, N. Ojima, K. Sato, M. Shiraishi, H. Shimizu, H. Nabeshima, S. Akazaki, K. Hori, and Y. Miyake, "Image-based skin color and texture analysis/ synthesis by extracting hemoglobin and melanin information in the skin," ACM Transactions on Graphic (TOG), pp. 770-779, 2003

[25] G. Burel, "Blind separation of sources: a non-linear neural algorithm," Neural Networks, vol. 5, pp. 937947, 1992.

[26] H. Shimazaki and S. Shinomoto, "A method for selecting the bin size of a time histogram," Neural Computation. vol. 19, no. 6, pp. 1503-1527, June 2007.

[27] D. G. Zhang and X. J. Kang, "A novel image denoising method based on spherical coordinates system," EURASIP Journal on Advances in Signal Processing, pp. 1-19, Jan. 2012.
[28] D. G. Zhang, G. Li, and K. Zheng, "An energybalanced routing method based on forward-aware factor for wireless sensor network," IEEE Transactions on Industrial Informatics, vol. 10, no. 1, pp. 766-773, Oct. 2014.

[29] D. G. Zhang, X. Wang, and X. D. Song, "A novel approach to mapped correlation of ID for RFID anticollision," IEEE Transactions on Services Computing, vol. 7, no. 4, pp. 741-748, Jul.2014.

[30] D. G. Zhang and Y. N, Zhu, "A new constructing approach for a weighted topology of wireless sensor networks based on local-world theory for the internet of things (IOT)," Computers \& Mathematics with Applications, vol. 64, no.5 pp. 1044-1055, Sep. 2012.

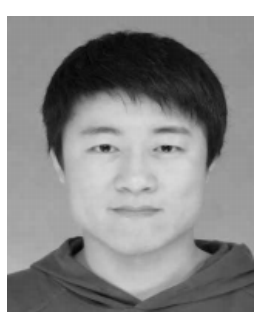

Liu Yang He received the B.S degrees in Software and Mechanical Engineering from Dalian Jiaotong University, China in 2010. He received the M.S degrees in IT Convergence and Application Engineering from Pukyong National University, Korea in 2012. $\mathrm{He}$ is currently a Ph.D. candidate in department of IT Convergence and Application engineering at the Pukyong National University. His research interests are in the area of digital image processing and radar retrieval.

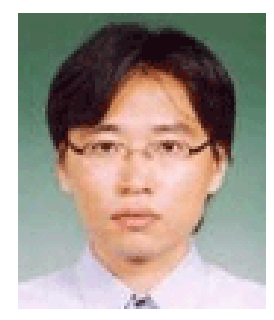

Suk-Hwan Lee He received his B.S., M.S., and Ph. D. degree in Electrical Engineering from Kyungpook National University, Korea in 1999, 2001, and 2004 respectively. He is currently an associate professor in Department of Information Security at Tongmyong University. His research interests include multimedia security, digital image processing, and computer graphics.

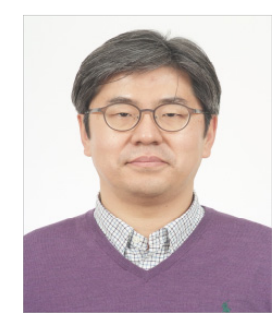

Seong-Geun Kwon He received hist B.S., M.S., and Ph. D. degrees in Electrical Engineering from Kyungpook National University, Korea in 1996, 1998, and 2002 respectively. $\mathrm{He}$ worked at Samsung Electronics as a senior researcher from 2002 to 2011. $\mathrm{He}$ is currently an associate professor in Department of Electronics Engineering at KyungIl University. His research interests include multimedia security, digital mobile broadcasting, and image processing. 


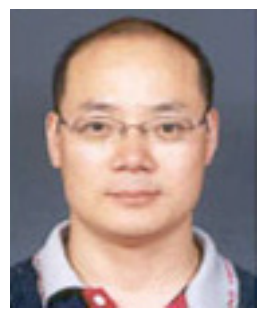

Ha-Joo Song He received his B.S., M.S., and Ph. D. degree in Computer Engineering from Seoul National University, Korea in 1993, 1995, and 2001 respectively. He was a principal technical staff of LimeTV Inc. He is currently a professor in Department of IT Convergence and Application Engineering at Pukyong National University. His research interests include object-oriented database, transaction processing and web-service.

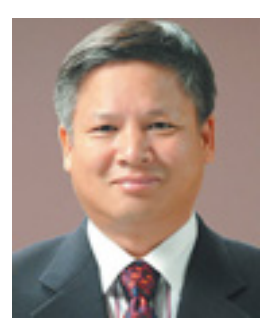

Ki-Ryong Kwon He received the B.S., M.S., and Ph.D. degrees in electronics engineering from Kyungpook National University in 1986, 1990, and 1994 respectively. He worked at Hyundai Motor Company from 1986-1988 and at Pusan University of Foreign Language from 1996-2006. He is currently a professor in Department of IT Convergence and Application Engineering at the Pukyong National University. He has researched University of Minnesota in USA on 2000-2002 with Post-Doc. and Colorado State University on 20112012 with visiting professor. He is currently the President of Korea Multimedia Society. His research interests are in the area of digital image processing, multimedia security and watermarking, bioinformatics, weather radar information processing. 